\section{Stimulus Control}

Joel W. Hughes

Department of Psychology, Kent State University, Kent, OH, USA

\section{Synonyms}

Conditioned stimulus

\section{Definition}

In classical or operant conditioning, stimulus control is present when the subject is trained so that the stimulus evokes the conditioned response or operant behavior. Stimulus control can increase or decrease the probability that the behavior will occur depending on the conditioning. Stimulus control is often used to establish discriminative stimuli so that the desired behavior occurs in the presence of a specific stimulus. Behavioral therapies based on stimulus control have been developed, such as stimulus control therapy for insomnia. These therapies seek to control behavior by conditioning patients to stimuli that act as antecedents to the desired behaviors.

\section{Cross-References}

Stimulus-Bound Behavior 